\title{
Miscarriage Rates Due To Endometriosis: A Retrospective Cohort Study
}

\author{
Pinar CALIS ${ }^{1}$, Gizem ISIK ${ }^{1}$, Dilara DUYGULU ${ }^{1}$, Nuray BOZKURT ${ }^{1}$, Deniz KARCAALTINCABA ${ }^{1}$ \\ Ankara, Turkey
}

\begin{abstract}
OBJECTIVE: To find the differences of miscarriage rates between pregnant patients with and without endometriosis.

STUDY DESIGN: The retrospective cohort study was conducted in the Department of Obstetrics and Gynecology, School of Medicine, Gazi University between January-2015 and December-2018. Patients were divided into two groups; endometriosis and non-endometriosis according to their pathology report and ultrasound examination. In both groups, miscarriage rates and in vitro fertilization pregnancy ratios were analyzed. Miscarriage rates in in vitro fertilization pregnancies and in endometriosis group who had and didn't have surgery also analyzed.
\end{abstract}

RESULTS: Sixty-two patients in the study group and 65 patients in the control group were included. There was no significant difference in miscarriage rates between endometriosis and non-endometriosis group (20/62 (32\%) and 13/65 (20\%), respectively, $p=0.124)$. There was a statistically significant difference in miscarriage rates between in vitro fertilization and non-in vitro fertilization group $(p=0.004)$. Apart from that, in in vitro fertilization group, the miscarriage rate was higher in the endometrioma subgroup than non-endometrioma $(p=0.008)$.

CONCLUSION: There was an only significant difference in miscarriage rate in the in vitro fertilization group between endometrioma and non-endometrioma patients. In the future, there should be more studies to define the actual pathophysiology for miscarriage with endometriosis.

Keywords: Endometrioma, Miscarriage, In vitro fertilization, Pregnancy

Gynecol Obstet Reprod Med 2021;27(2):128-131

\section{Introduction}

Endometriosis is a frequent and one of the most important benign gynecologic disease in reproductive age. It is seen $10 \%$ of women in general population and $40 \%$ in women with subfertility $(1,2)$. The main symptoms in endometriosis are pelvic

\footnotetext{
${ }^{1}$ Gazi University Faculty of Medicine, Department of Obstetrics and Gynecology, 06560, Ankara, Turkey

Address of Correspondence: Pinar Calis

Gazi University Faculty of Medicine

Department of Obstetrics and Gynecology,

06560, Ankara, Turkey

ptokdemir86@gmail.com
}

Submitted for Publication: 16.12.2019 Revised for Publication: 7.01.2020 Accepted for Publication: 20.02.2020 Online Published: 02.08.2021

ORCID IDs of the authors: $\quad$ PC: 0000-0001-9334-1987

GI: 0000-0001-8810-3781 DD: 0000-0001-9983-2306

NB: 0000-0002-1107-9629

DK: 0000-0001-5276-9303

\begin{tabular}{|c|c|}
\hline Quick Response Code: & Access this article online \\
\cline { 2 - 2 } & $\begin{array}{c}\text { Website: www.gorm.com.tr } \\
\text { e- mail: info@gorm.com.tr }\end{array}$ \\
\cline { 2 - 3 } & DOI:10.21613/GORM.2020.1058 \\
\hline
\end{tabular}

How to cite this article: Calis P. Isik G. Duygulu D. Bozkurt N. Karcaaltincaba D. Miscarriage Rates Due To Endometriosis: A Retrospective Cohort Study. Gynecol Obstet Reprod Med 2021;27(2):127-131 pain and infertility. Even though endometriosis causes infertility by interrupting whole reproductive process such as oocyte-sperm interaction, ovarian reserve and implantation, by the developments in assisted reproductive technology (ART) treatments, pregnancy rates in patients with endometriosis have increased $(3,4)$.

Miscarriage is spontaneous loss of a fetus before 20 gestational weeks. Miscarriage is the most frequently seen pregnancy complication in the first trimester (5). Although traditionally, pregnancy has positive effect on endometriosis and its symptoms (6), endometriosis has opposite effects on pregnancy. Recent studies showed that eutopic endometrium in patients with endometriosis was unsuitable for implantation (7). By this way, decidualized stroma and impaired placentation cause miscarriages (8). However, the results were conflicting that, there were several studies that showed similar miscarriage rates between patients with and without endometriosis (9). On the other hand, because patients with endometriosis mostly become pregnant with In Vitro Fertilization (IVF) techniques, this could also affect rates of miscarriages of patients.

In the current study, our aim is to compare miscarriage rates in patients with and without endometriosis. Miscarriage 
rates between IVF and non-IVF patients with endometriosis will also be compared.

\section{Material Method}

\section{Study Design}

This retrospective cohort study was conducted in the Department of Obstetrics and Gynecology, School of Medicine, Gazi University between January-2015 and December-2018 from our electronic database. In our outpatient clinic, pregnant patients with known endometriosis (had surgery or not) and without endometriosis were recruited. The inclusion criteria were (1) females aged between 18-45, (2) patients with known endometriosis with ultrasound examination or pathology report (case group) and (3) patients who were followed up in our clinic from the first trimester. To determine endometriosis with ultrasound examination, the patient should have had at least two positive ultrasound findings for endometriosis apart from 3 months. These findings were round-shaped cystic mass with homogeneous low echogenic fluid content with scattered internal echoes (10). The exclusion criteria were (1) maternal systematic diseases which could cause miscarriage (rheumatologic, diabetes, kidney, etc.), (2) abnormal karyotype of women or her husband, (3) known abnormal uterine cavity (presence of submucosal fibroids) or uterine malformations, (4) quit pregnancy follow up in our clinic. For the control group, from the same period time, pregnant patients who did not have endometriosis but with similar demographic properties as the endometriosis group was formed. Randomization was made for selecting control group patients. Approval from an institutional review board was obtained (Approval No: 24/12/2018-942).

In both groups, miscarriage rates, and IVF pregnancy ratios were analyzed. In addition to that, miscarriage rates were analyzed in patients with endometriosis who had surgery or not.

\section{Statistical Analysis}

The data analysis was performed by SPSS 24.0 software (IBM Corporation, Armonk, NY, USA). The continuous variables were analyzed using the Kruskal-Wallis test, and the categorical variables using the Pearson chi-square test. $p<0.05$ was considered significant.

\section{Results}

Of the 3438 women that had been followed up in our clinic in the study period, 62 patients had endometrioma and were appropriate for the final evaluation according to inclusion and exclusion criterion as a study group. 65 patients were formed as a control group randomly without endometrioma with similar demographic properties with the study group (Figure 1). The mean female ages in the endometriosis and non-endometriosis groups were $33 \pm 0.61$ and $31 \pm 0.54$ years, respectively (Table I).

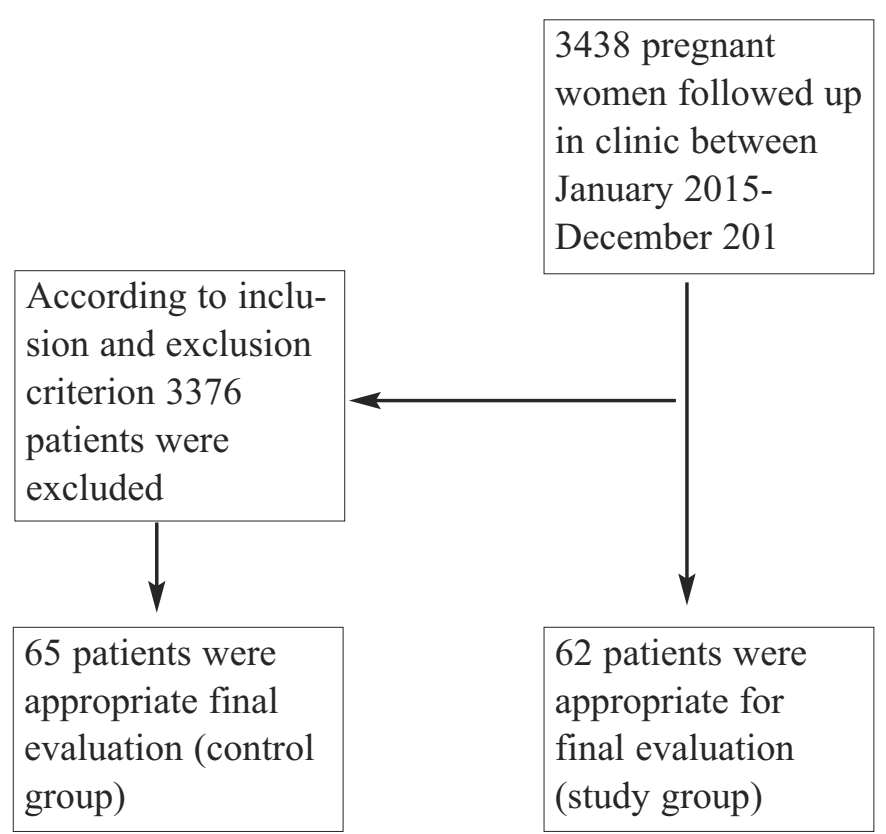

Figure 1: Selection of study and control group patients according to inclusion and exclusion criterion.

Table I: Demographic properties and $p$ values of two groups.

\begin{tabular}{|c|c|c|c|}
\hline Characteristics & $\begin{array}{l}\text { Endometrioma } \\
(\mathrm{n}=62)\end{array}$ & $\begin{array}{l}\text { Control } \\
(n=65)\end{array}$ & $\begin{array}{l}p \\
\text { value }\end{array}$ \\
\hline Age (years) & $33 \pm 0.61$ & $31 \pm 0.54$ & $0.002^{*}$ \\
\hline BMI (kg/m2) & $22.91 \pm 3.12$ & $23.83 \pm 2.46$ & 0.16 \\
\hline Gravidity [n(\%)] & & & 0.23 \\
\hline None & $31(50)$ & $32(49.2)$ & \\
\hline 1 & $18(29)$ & $20(30.8)$ & \\
\hline 2 & $12(19.4)$ & $11(16.9)$ & \\
\hline$\geq 3$ & $1(1.6)$ & $2(3.1)$ & \\
\hline Parity [n(\%)] & & & 0.27 \\
\hline None & $53(85.5)$ & $55(84.6)$ & \\
\hline 1 & $8(12.9)$ & $8(12.4)$ & \\
\hline 2 & $1(1.6)$ & $2(3)$ & \\
\hline IVF [n(\%)] & & & 0.06 \\
\hline+ & $24(38.7)$ & $9(13.8)$ & \\
\hline- & $38(61.3)$ & $56(86.2)$ & \\
\hline
\end{tabular}

${ }^{*} p<0.05$ is thought statistically significant.

In Vitro Fertilization pregnancy ratios in study and control groups are also similar $(p=0.06)$. The miscarriage rates between patients with endometriosis and without endometriosis were 20/62 (32\%) and 13/65 (20\%), respectively ( $p=0.124)$. From the data, if a patient had endometrioma, there was a statistically significant difference in miscarriage rates between IVF and non-IVF group ( $52.4 \%$ vs $10 \%, p=0.004)$. Apart from that, in the IVF group, if a patient had endometrioma the miscarriage rate was higher if the patient did not have endometrioma $(p=0.008)$.

In addition, for endometrioma group, $56.9 \%$ of patients had surgery and $43.1 \%$ had diagnosed with ultrasound. There 
was no statistical difference in miscarriage rates if the patient had surgery for endometrioma or not $(p=0.385)$.

\section{Discussion}

The study shows that in patients with endometrioma, higher miscarriage rates were detected if they had IVF compared with non-IVF ones. On the other hand, according to data, miscarriage rates were not statistically significant between patients with endometrioma and without endometrioma. However, between IVF group patients, there was a statistically significant difference in miscarriage rates between endometriosis and non-endometriosis group.

In 2015, 10.8 million people were affected globally from endometriosis (11). Although it is seen most commonly between the ages of thirty and forty, it could be seen as early as eight years old $(12,13)$. Besides pelvic pain, endometriosis not only causes infertility but also could be a reason for adverse obstetric outcomes, especially for miscarriages. The main pathophysiology under this result could be thought that endometriosis causes loss of differentiation capacity of endometriotic cell lines. This forms a eutopic endometrium which is unsuitable for implantation (7).

Several observational nonrandomized studies have conflicting results for endometriosis and miscarriage. In a recent study in 2019 by Farland et al, 196,722 pregnant patients with and without endometriosis were examined. They found stillbirth, preterm birth, low birth weight, preeclampsia and also miscarriage rates (RR 1.40, 95\% CI 1.31-1.49) were higher in the endometriosis group (14). In addition to that, Saraswat et al. performed a national population-based cohort study and found that miscarriage rates were higher in women with endometriosis compared to women without endometriosis (OR $1.76,95 \%$ CI 1.44-2.15) (15).

On the other hand, in a meta-analysis, Hamdam et al compared the ART results according to miscarriages in patients with endometriosis and non-endometriosis. There was no statistically significant difference in miscarriage rates between groups (16). In a recent study by Yang et al, they collected women who went to the IVF cycle and divided them into endometriosis and non-endometriosis group. The study couldn't find any statistical difference between two groups in miscarriage rates (17). However, in our study, in IVF group we found a statistically significant difference between the endometriosis and non-endometriosis group. Besides, we compared also the IVF and non-IVF groups for miscarriage rates. And we could only find the statistically significant difference in miscarriage rates between patients with endometrioma in IVF and non-IVF group.

For proving this eutopic endometrium, one of the major study in literature was published by Hauzman et al in 2013 (18). In this study, they split the oocytes from the same healthy donors to patients with and without endometriosis. The results suggested that endometrial receptivity was not affected by stage III/IV endometriosis. Although there were studies in the literature that supported that patients with endometriosis had peritoneal fluid and serum that contained abnormal levels of factors such as tissue inhibitor of metalloproteinase-1 $(19,20)$, by this prospective study this hypothesis were not supported anymore.

In our study, the main reasons for higher miscarriage rates in IVF group could be first of all, in IVF patients there was a close pregnancy follow up. In this way, almost all miscarriages could be detected, but in non-IVF group some miscarriages, especially in early miscarriages, could not be diagnosed. In addition to that, without endometrioma, the spontaneous miscarriage rate was higher in the IVF group. Besides, if the IVF group was evaluated for miscarriage, miscarriage rate was higher in patients with endometrioma. This finding supported the eutopic endometrium hypothesis, which increased the miscarriage rates.

The main limitation of the study is the sample size. Although the last three years were analyzed in the current study, by increasing the sample size there could be more statistically significant differences could be found between groups.

As a result, endometriosis is one of the most seen gynecologic problems which affects fertility and pregnancy outcomes. Although there are several hypotheses about miscarriage rates and endometriosis, we could only find a significant difference in miscarriage in IVF group between endometriosis and non-endometriosis patients. In the future, there should be some basic studies to define the actual pathophysiology for miscarriage with endometriosis.

\section{Acknowledgements: None \\ Funding: None}

Disclosure: The authors have no conflicts of interest to declare.

Author Contribution: PC: Design, statistics, manuscript writing, GI: Collecting data, manuscript writing, DD: Collecting data, NB: Editing manuscript, DK: Statistics, editing manuscript.

Consent for using data was taken.

The study was conducted in accordance with the Declaration of Helsinki

\section{References}

1. Giudice LC. Clinical practice. Endometriosis. N Engl J Med. 2010;362(25):2389-98. doi: 10.1056/NEJMcp1000 274.

2. Ozkan S, Arici A. Advances in treatment options of endometriosis. Gynecol Obstet Invest. 2009;67(2):81-91. doi: 10.1159/000163071. 
3. Gupta S, Goldberg JM, Aziz N, Goldberg E, Krajcir N, Agarwal A. Pathogenic mechanisms in endometriosis-associated infertility. Fertil Steril. 2008;90(2):247-57. doi: 10.1016/j.fertnstert.2008.02.093.

4. de Ziegler D, Borghese B, Chapron C. Endometriosis and infertility: pathophysiology and management. Lancet. 2010;376(9742):730-8. doi: 10.1016/S0140-6736(10)604 90-4.

5. Nybo Andersen AM, Wohlfahrt J, Christens P, Olsen J, Melbye M. Maternal age and fetal loss: population based register linkage study. BMJ. 2000;320(7251):1708-12. doi: 10.1136/bmj.320.7251.1708.

6. Brosens I, Brosens JJ, Fusi L, Al-Sabbagh M, Kuroda K, Benagiano G. Risks of adverse pregnancy outcome in endometriosis. Fertil Steril. 2012;98(1):30-5. doi: 10.1016/j. fertnstert.2012.02.024.

7. Klemmt PA, Carver JG, Kennedy SH, Koninckx PR, Mardon HJ. Stromal cells from endometriotic lesions and endometrium from women with endometriosis have reduced decidualization capacity. Fertil Steril. 2006;85 doi: 10.1016/j.fertnstert.2005.08.046.(3):564-72.

8. Matsuzaki S, Schubert B. Oxidative stress status in normal ovarian cortex surrounding ovarian endometriosis. Fertil Steril. 2010;93(7):2431-2. doi: 10.1016/j.fertnstert. 2009. 08.068 .

9. Leonardi M, Papaleo E, Reschini M, Pagliardini L, Benaglia L, Candotti G, et al. Risk of miscarriage in women with endometriosis: insights from in vitro fertilization cycles. Fertil Steril. 2016;106(2):386-92 e3. doi: 10.1016/j.fertnstert.2016.03.047.

10. Savelli L. Transvaginal sonography for the assessment of ovarian and pelvic endometriosis: how deep is our understanding? Ultrasound Obstet Gynecol. 2009;33(5):497501. Doi: 10.1002/uog.6392.

11. GBD 2015 Disease and Injury Incidence and Prevalence Collaborators. Global, regional, and national incidence, prevalence, and years lived with disability for 310 diseases and injuries, 1990-2015: a systematic analysis for the Global Burden of Disease Study 2015. Lancet. 2016;
388(10053):1545-602. doi: 10.1016/S0140-6736(16)316 78-6.

12. Endometriosis. 2017; Available from: womenshealth.gov.

13. McGrath PJ SB, Walker SM, Zempsky WT. Oxford Textbook of Paediatric Pain. 2013.

14. Farland LV, Prescott J, Sasamoto N, Tobias DK, Gaskins AJ, Stuart JJ, et al. Endometriosis and risk of adverse pregnancy outcomes. Obstet Gynecol. 2019;134(3):52736. doi: 10.1097/AOG.0000000000003410.

15. Saraswat L, Ayansina DT, Cooper KG, Bhattacharya S, Miligkos D, Horne AW, et al. Pregnancy outcomes in women with endometriosis: a national record linkage study. BJOG. 2017;124(3):444-52. doi: 10.1111/14710528.13920 .

16. Hamdan M, Omar SZ, Dunselman G, Cheong Y. Influence of endometriosis on assisted reproductive technology outcomes: a systematic review and meta-analysis. Obstet Gynecol. 2015;125(1):79-88. doi: 10.1186/s12958019-0463-1.

17. Yang P, Wang Y, Wu Z, Pan N, Yan L, Ma C. Risk of miscarriage in women with endometriosis undergoing IVF fresh cycles: a retrospective cohort study. Reprod Biol Endocrinol. 2019;17(1):21. doi: 10.1186/s12958-0190463-1.

18. Hauzman EE, Garcia-Velasco JA, Pellicer A. Oocyte donation and endometriosis: What are the lessons? Semin Reprod Med. 2013;31(2):173-7. doi: 10.1055/s-0032-133 3483.

19. Sharpe-Timms KL, Keisler LW, McIntush EW, Keisler DH. Tissue inhibitor of metalloproteinase-1 concentrations are attenuated in peritoneal fluid and sera of women with endometriosis and restored in sera by gonadotropinreleasing hormone agonist therapy. Fertil Steril. 1998; 69(6):1128-34. doi: 10.1016/s0015-0282(98)0007 5-2.

20. Taketani Y, Kuo TM, Mizuno M. Comparison of cytokine levels and embryo toxicity in peritoneal fluid in infertile women with untreated or treated endometriosis. Am J Obstet Gynecol. 1992;167(1):265-70. doi: 10.1016/s00 02-9378(11)91672-x. 